\title{
Single-wavelength reflected confocal and multiphoton microscopy for tissue imaging
}

\author{
Wei-Liang Chen* \\ Chen-Kuan Chou* \\ Ming-Gu Lin* \\ Yang-Fang Chen \\ National Taiwan University \\ Department of Physics \\ No. 1 Section 4 Roosevelt Road \\ Taipei 10617, Taiwan
}

\section{Shiou-Hwa Jee}

National Taiwan University Hospital

Department of Dermatology

No. 7 Chung-Shan South Road

Taipei 100, Taiwan and

National Taiwan University College of Medicine

Department of Dermatology

No 1 Jen Ai Road Section 1, Taipei 100, Taiwan

\section{Hsin-Yuan Tan}

National Taiwan University

Institute of Biomedical Engineering

Taipei 100, Taiwan and

Chang Gung Memorial Hospital

Department of Ophthalmology

No. 5 Fu-Shin Street

Tao Yuan 333, Taiwan

\section{Tsung-Hua Tsai}

Far Eastern Memorial Hospital

Department of Dermatology

No. 21 Nan-Ya South Road Section 2 Pan-Chiao

Taipei 200, Taiwan

\section{Ki-Hean Kim \\ Daekeun Kim \\ Peter T. C. So}

Massachusetts Institute of Technology

Department of Mechanical Engineering and

Department of Biological Engineering

Cambridge, Massachusetts 02139

\author{
Sung-Jan Lin $^{\dagger}$ \\ National Taiwan University Hospital \\ Department of Dermatology \\ No. 7 Chung San South Road \\ Taipei 100, Taiwan \\ and \\ National Taiwan University \\ Institute of Biomedical Engineering \\ No. 1 Section 1 Jen-Ai Road \\ Taipei 100, Taiwan
}

\footnotetext{
*These authors contributed equally to this work.

${ }^{+}$Address all correspondence to: Sung-Jan Lin, National Taiwan University Hospital, Department of Dermatology, No. 7 Chung San South Road, Taipei 100, Taiwan; E-mail: drsjlin@ntu.edu.tw; and Chen-Yuan Dong, National Taiwan University, Department of Physics, No. 1 Section 4 Roosevelt Road, Taipei 10617, Taiwan; Tel: +886-2-33665155; Fax: +886-2-33665244; E-mail: cydong@phys.ntu.edu.tw
}

\author{
Chen-Yuan Dong ${ }^{\dagger}$ \\ National Taiwan University \\ Department of Physics \\ and \\ Center for Quantum Science and Engineering \\ Taipei 10617, Taiwan
}

\begin{abstract}
Both reflected confocal and multiphoton microscopy can have clinical diagnostic applications. The successful combination of both modalities in tissue imaging enables unique image contrast to be achieved, especially if a single laser excitation wavelength is used. We apply this approach for skin and corneal imaging using the 780-nm output of a femtosecond, titanium-sapphire laser. We find that the near-IR, reflected confocal (RC) signal is useful in characterizing refractive index varying boundaries in bovine cornea and porcine skin, while the multiphoton autofluorescence (MAF) and second-harmonic generation (SHG) intensities can be used to image cytoplasm and connective tissues (collagen), respectively. In addition, quantitative analysis shows that we are able to detect MAF from greater imaging depths than with the near-IR RC signal. Furthermore, by performing RC imaging at 488, 543, and $633 \mathrm{~nm}$, we find that a longer wavelength leads to better image contrast for deeper imaging of the bovine cornea and porcine skin tissue. Finally, by varying power of the 780-nm source, we find that comparable RC image quality was achieved in the 2.7 to $10.7-\mathrm{mW}$ range. $\odot 2009$ Society of Photo-Optical Instrumentation Engineers. [DOI: 10.1117/1.3247157]
\end{abstract}

Keywords: multiphoton fluorescence; second-harmonic generation; reflected confocal imaging; optical microscopy.

Paper 08222RRR received Jul. 10, 2008; revised manuscript received Aug. 11, 2009; accepted for publication Aug. 11, 2009; published online Oct. 26, 2009.

\section{Introduction}

There has been considerable recent interest in applying optical microscopy imaging techniques for the minimally invasive, label-free imaging and diagnosis of pathological tissues. In the submicrometer resolution range, two techniques independently emerged as promising candidates in tissue diagnosis. The development of reflected confocal (RC) microscopy based on reflection from interfaces of different refractive indices achieved enormous success in both dermatology and ophthalmology. Although the RC signal lacks molecular specificity, tissue abnormalities and pathogens can often be identified with this modality. ${ }^{1-4}$ In comparison, multiphoton (MP) tissue microscopy achieves image contrast by inducing nonlinear optical phenomena such as fluorescence excitation or harmonic generation from the specimens of interests. ${ }^{5,6}$ While RC and MP microscopy share common features such as the ability to achieve excellent axial depth discrimination and

1083-3668/2009/14(5)/054026/8/\$25.00 ○ 2009 SPIE 


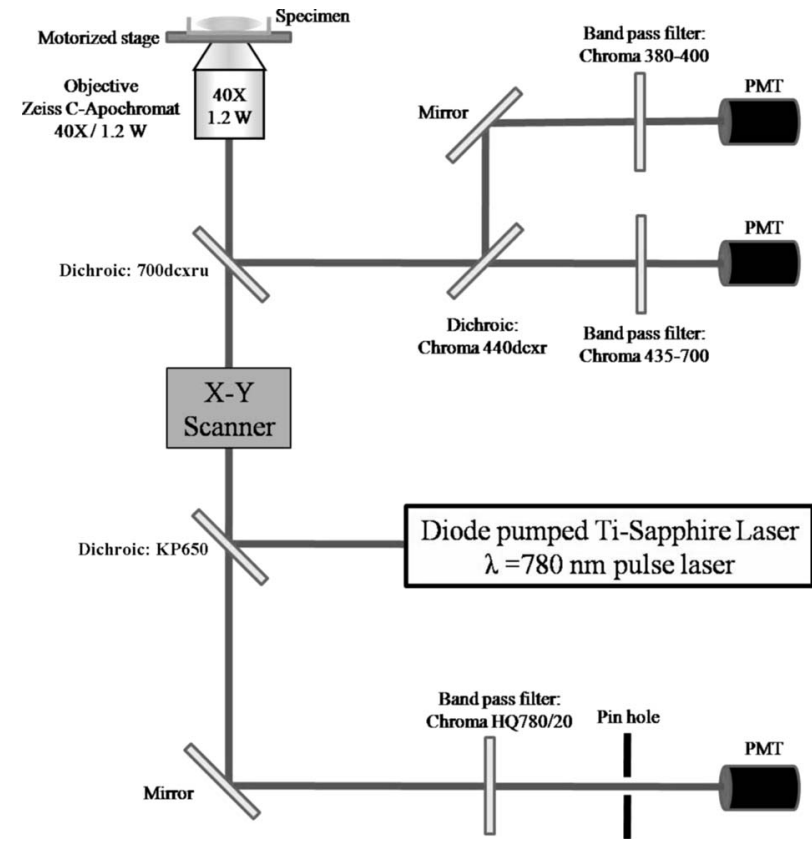

Fig. 1 Experimental arrangement for the single-excitation wavelength, RC, and multiphoton imaging system.

label-free imaging, the fundamental differences of the image contrast mechanisms dictate the choice of the imaging modality in a particular application. Specifically, while the RC signal is capable of detecting the presence of interfaces with different refractive indices, multiphoton autofluorescence (MAF) and second-harmonic generation (SHG) intensities can be used for the respective monitoring of cellular metabolism and the imaging of noncentrosymmetric biological structures such as collagen and muscle fibers. ${ }^{7,8}$

In tissue imaging, multiphoton microscopy has been well demonstrated in imaging both the skin and ocular surface. ${ }^{9-11}$ The ease of accessing these two tissues enables them to be the preferred choices for optical imaging diagnostics. Furthermore, multiphoton fluorescence and SHG microscopy have proven to be effective in discriminating pathological tissues from their normal counterparts in a wide array of dermato- logical and ophthalmological conditions including skin aging, basal cell carcinoma, laser ablation effects, keratoconus, and corneal infection. ${ }^{12-17}$

However, in most applications, tissue imaging is achieved with either RC or MP microscopy. In the few reports in which both modalities are implemented, two wavelengths from different excitation sources were used for the independent detection of the RC and MP signals. ${ }^{18}$ Clearly, the ability to achieve both RC and MP microscopy with a single excitation wavelength will be significant for tissue imaging in several ways. First, the multimodal information of the RC, MAF, and SHG signals offers researchers or clinicians additional information in tissue diagnosis. Second, the use of a single wavelength for specimen examination avoids artifacts associated with chromatic aberration. Specifically, the issue associated with axial image shift from chromatic aberration can be avoided. Finally, the use of a near-IR source to obtain the RC signal can avoid possible photodamage from visible sources. However, since the refractive index of a material tends to decrease with increasing wavelengths, there is a concern whether the near-IR, $\mathrm{RC}$ signal from tissues is sufficient for imaging purposes.

In this paper, we demonstrate the combination of RC, MAF, and SHG imaging on ex vivo bovine cornea and porcine skin specimens using the single excitation wavelength of $780 \mathrm{~nm}$ from a femtosecond, titanium-sapphire laser. In addition, we also investigate the effects of different excitation wavelengths $(488,543$, and $633 \mathrm{~nm})$ on RC imaging. The effects of varying the power of $780 \mathrm{~nm}$ for RC imaging are also examined.

\section{Materials and Methods}

The combined RC and MP microscope used in this study is based on a commercial laser scanning microscope (LSM 510 Meta, Zeiss, Germany). As shown in Fig. 1, a diode-pumped, solid state (DPSS) laser (Millennia X, Spectra-Physics, Mountain View) pumped titanium-sapphire source (Tsunami, Spectra Physics) with the output wavelength of $780 \mathrm{~nm}$ was used as the excitation source. The laser beam was first reflected by a short-pass dichroic mirror (KP650) before entering the scanning mirror unit. The scanned laser beam then passes through a long pass dichroic mirror (700dcxru, Chroma Technology, Rockingham, Vermont) and into the ob-

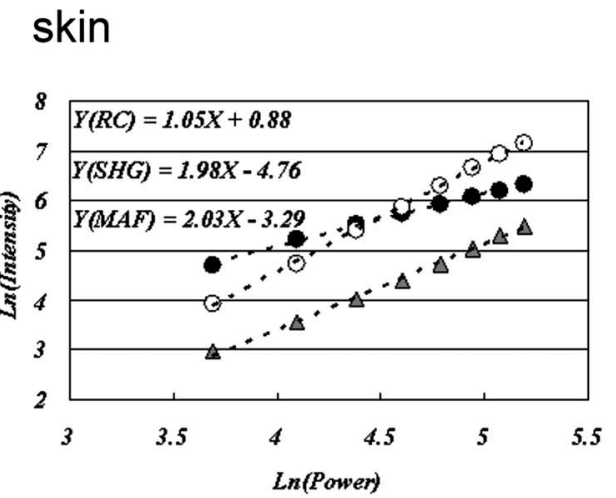

(a)

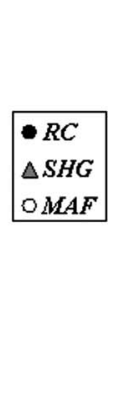

$\triangle S H G$ $M A F$

Fig. 2 Power dependence of the RC, MAF, and SHG signals in the (a) porcine skin and (b) bovine cornea specimen. 


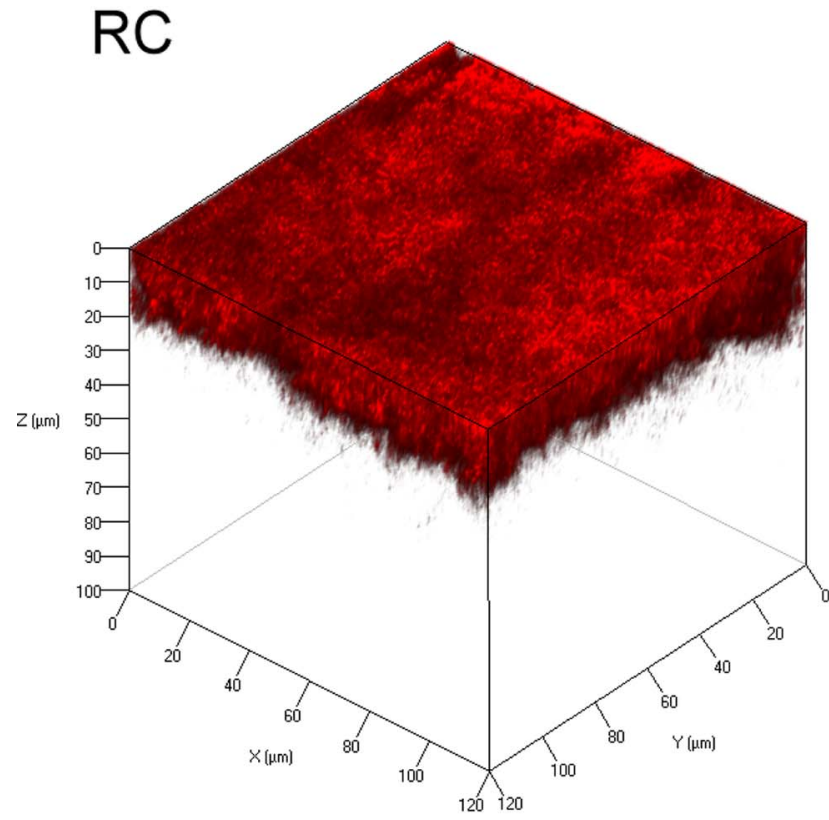

(a)

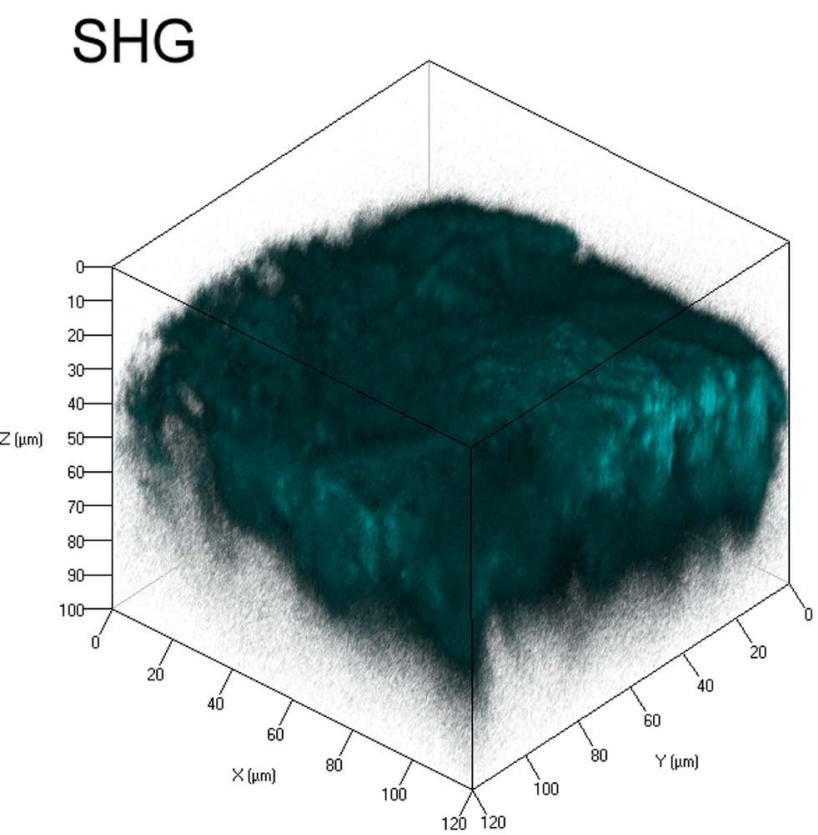

(c)

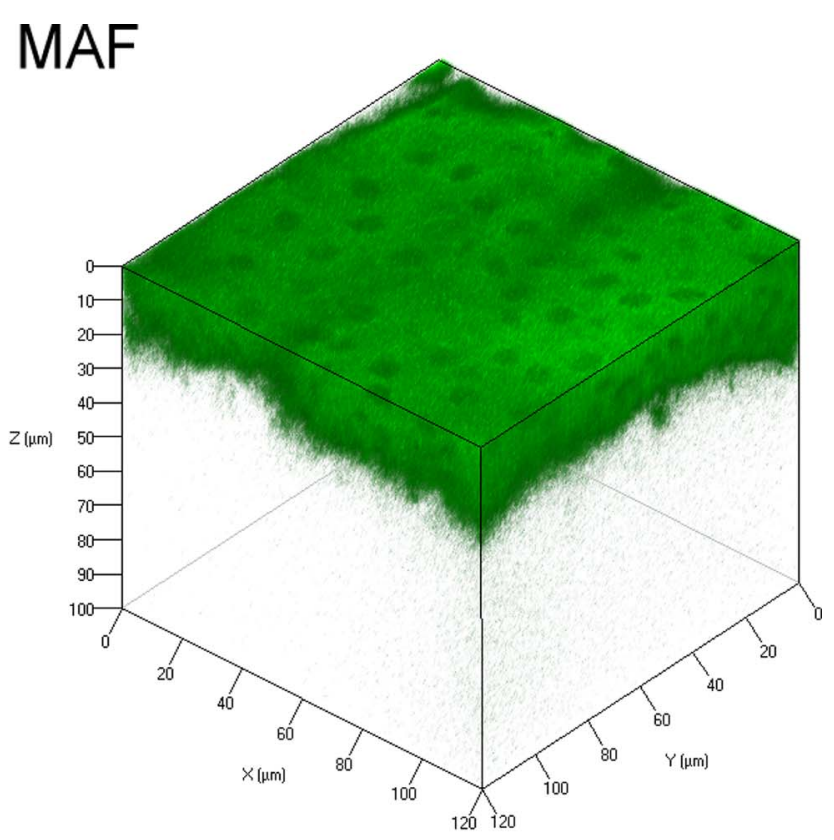

(b)

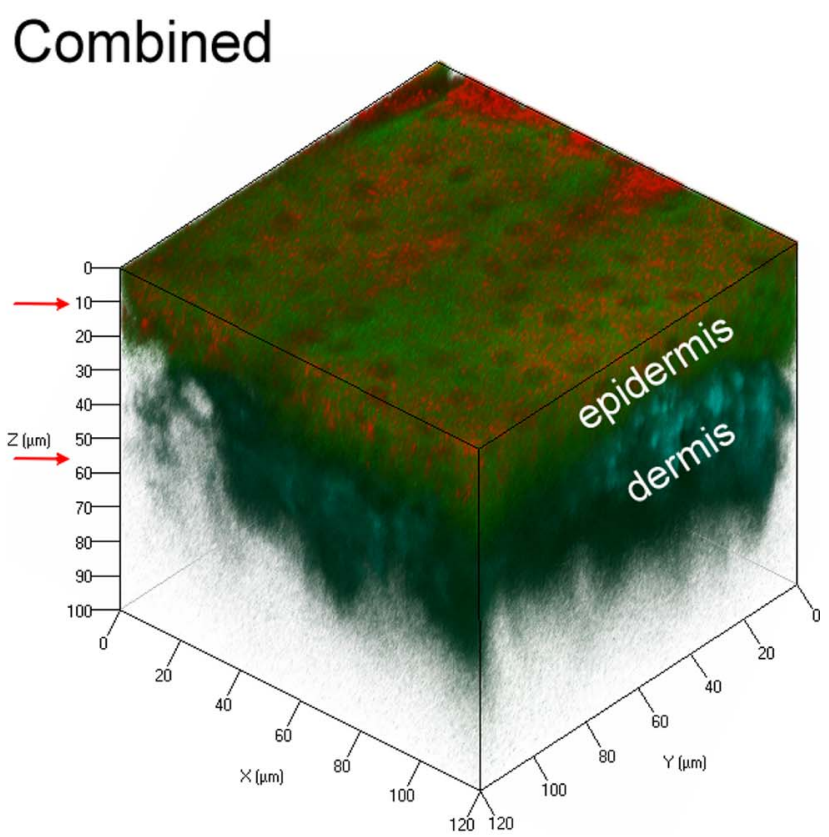

(d)

Fig. 3 (a) RC (red), (b) MAF (green), (c) SHG (cyan), and (d) combined RC, MAF, and SHG imaging of the ex vivo human skin specimen in 3-D. Image sizes are $115 \times 115 \times 100 \mu \mathrm{m}^{3}$. (Color online only.)

jective. For imaging purposes, we used a high-resolution, water-immersion objective [C-Apochromat 40×, numerical aperture (NA) 1.2, Zeiss] to focus the excitation source onto the specimen and the collection of RC, epi-illuminated autofluorescence (AF), and backward SHG signals from the specimens. For multiphoton imaging, the signal were acquired using the nondescanned detection in which the MAF and SHG signals were reflected by the 700dcxru dichroic mirror and further separated by the combination of a secondary dichroic mirror (440 dcrxu, Chroma Technology) and two bandpass filters (e700sp-2p-e435, HQ390/22m-2p, Chroma Technol- ogy). The detection bandwidths of the MAF and SHG signals were 435 to 700 and 380 to $400 \mathrm{~nm}$ respectively. For RC imaging, the signal were detected in the descanned mode where the reflected 780-nm light was descanned and passed through the KP650 dichroic mirror, the bandpass filter (HQ780/20, Chroma Technology), and the confocal pinhole before detection by the photomultiplier tube (PMT). Although KP650 is a short-pass filter, the reflected signal was sufficiently intense to provide good signal registration. 
To accommodate the capabilities of the Zeiss system, the descanned $\mathrm{RC}$ and nondescanned multiphoton image acquisition were sequentially acquired in the two-pass mode. The use of a single laser wavelength and the same filter and dichroic arrangement for both the RC and MP imaging demonstrates the feasibility of achieving image contrast by different mechanisms in simultaneous acquisition. The use of the two-pass approach is dictated by the Zeiss scanning software, which prevents the simultaneous acquisition of both the descanned and nondescanned signals. Finally, the pixel dwell time was $1.6 \mu \mathrm{s}$, and each image was average over 16 repeated scans for each of the two imaging modes. For the 512 $\times 512$ pixels per frame corresponding to a frame size of $115.17 \times 115.17 \mu \mathrm{m}^{2}$, each image in each mode was acquired in $6.71 \mathrm{~s}$. For the ex vivo bovine cornea and porcine skin specimens, the on sample power of the 780-nm source was $10 \mathrm{~mW}$. The same laser power settings were used in both the descanned RC and nondescanned MP imaging. In addition, to evaluate the effectiveness of different wavelengths for RC imaging, we also imaged porcine skin and bovine cornea samples using the 488-, 543-, and 633-nm laser sources in the Zeiss LSM 510 system. In studies using the visible sources, each image was produced using an on-sample power of around $0.03 \mathrm{~mW}$, and the detector gain was independently optimized for each case.

To compare the signal strength at different imaging depths, we averaged the pixel intensity for each imaging modality over the acquired image at each depth, and normalized the resultant value with respect to the maximum observed value over the range of imaging depths.

Both the ex vivo bovine corneal and porcine skin specimens were used in this study. Bovine eyes were purchase from a local meat shop. After removing excessive tissue around the bovine eyes, the specimens were placed in a dish with a cover glass bottom. With the cornea in contact with the cover glass, phosphate buffer solution (PBS) was added to simulate physiological conditions for imaging of the eye specimen. The ex vivo porcine skin specimen used in this study was also obtained from a local meat shop. The skin specimen was placed in a well slide, filled with PBS, and covered with a cover glass for viewing.

\section{Results and Discussion}

To verify that the RC, MAF, and SHG processes correspond to either the one- or multiphoton processes, we measured the signal levels as a function of excitation power in both specimens. The results, plotted on the natural logarithmic scale, are shown in Figs. 2(a) and 2(b) for skin and cornea. As the results indicate, the slopes of the RC, MAF, and SHG signals are, respectively, 1.05, 2.03, and 1.98 for skin and 1.05, 1.96, 1.92 for cornea. These observations demonstrate that our system is effective in detecting and separating the one-photon RC signal from the multiphoton signals.

The RC and MP images of ex vivo porcine skin and bovine cornea are shown in Figs. 3-6. In all images, the pseudocolors of red, green, and cyan represent the RC, AF, and SHG signals, respectively. Figure 3 shows the 3-D image stack of the skin specimen to a depth of $100 \mu \mathrm{m}$ and the detailed 2-D images at the selected depths of 10 and $55 \mu \mathrm{m}$, corresponding to the two marked depths [red arrow in Fig. 3(d)], are shown

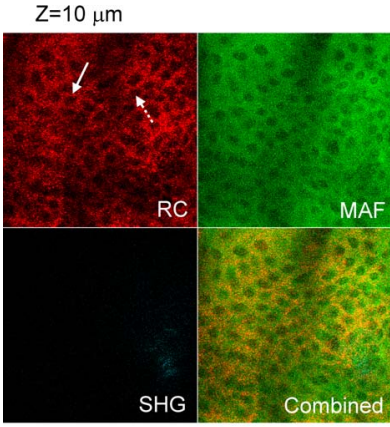

(a)

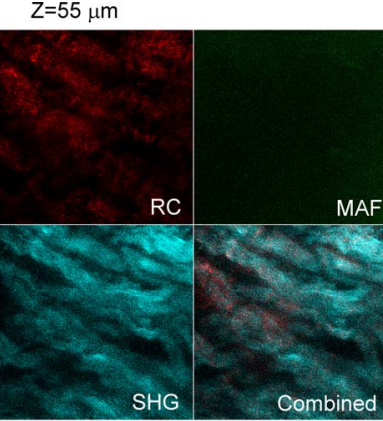

(b)
Fig. 4 RC (red), MAF (green), SHG (cyan), and combined RC, MAF, and SHG 2-D images of the ex vivo human skin specimen at the approximate imaging depths of (a) $10 \mu \mathrm{m}$ and (b) $55 \mu \mathrm{m}$. Image sizes are $115 \times 115 \mu \mathrm{m}^{2}$. (Color online only.)

in Fig. 4. The 3-D stack and 2-D images reveal that the near-IR RC signal is most effective in outlining the epithelial layers, while the AF is effective for cytoplasmic imaging of the epidermis. Specifically, at the depths of $10 \mu \mathrm{m}$, we were able to observe the cellular boundaries (white arrows) and the nuclear regions (white dashed arrows), suggesting that significant refractive index changes in the near-IR range, in the skin epithelium, occurs at the intercellular and nuclear boundaries. Furthermore, note that the RC signal can also be used to delineate the cytoplasmic regions, suggesting the presence of a heterogeneous distribution of refractive index varying organelles there. Finally, the SHG signal is found only in the dermal layers, corresponding to the presence of collagen fibers.

Since the cornea is another organ in which reflected confocal microscopy has been successfully applied, ${ }^{3,4}$ we also tested our multimodal imaging modality on ex vivo bovine cornea specimens. Similar to our presentation of the skin results, we show the 3-D corneal image stack in Fig. 5, and the detailed imaged at selected depths of 30 (epithelium) and $160 \mu \mathrm{m}$ (stroma) are shown in Fig. 6. Similar to the skin results, in the corneal epithelium, the $\mathrm{RC}$ signal is effective for outlining the individual epithelial cells (white arrow at $z$ $=30 \mu \mathrm{m}$ ) and their nuclei (white dashed arrow at $z=30 \mu \mathrm{m}$ ), while the AF signal can be used for identifying the cytoplasmic regions. However, unlike skin imaging, the RC signal is effective only for identifying epithelial cellular boundaries and the nuclear regions with insignificant RC signal detected from within the cytoplasmic domains. This may be due to the higher water content of the corneal epithelium, which minimized refractive index varying boundaries in corneal epithelium. Within the stroma, SHG imaging reveals the architecture of the collagen matrix while individual keraotcytes can be traced using the near-IR, RC signal (white arrow $z$ $=160 \mu \mathrm{m})$.

To further demonstrate the achievable imaging depths of the RC, MAF, and SHG signals, in Fig. 7 we plot the detected $\mathrm{RC}, \mathrm{MAF}$, and SHG signals for the porcine skin and bovine cornea specimens found in Figs. 3 and 5. The three signals were computed at each depth. From the result, we can see that the near-IR, RC signal is effective for imaging the bovine cornea and porcine skin specimens to depths of approximately 
$\mathrm{RC}$

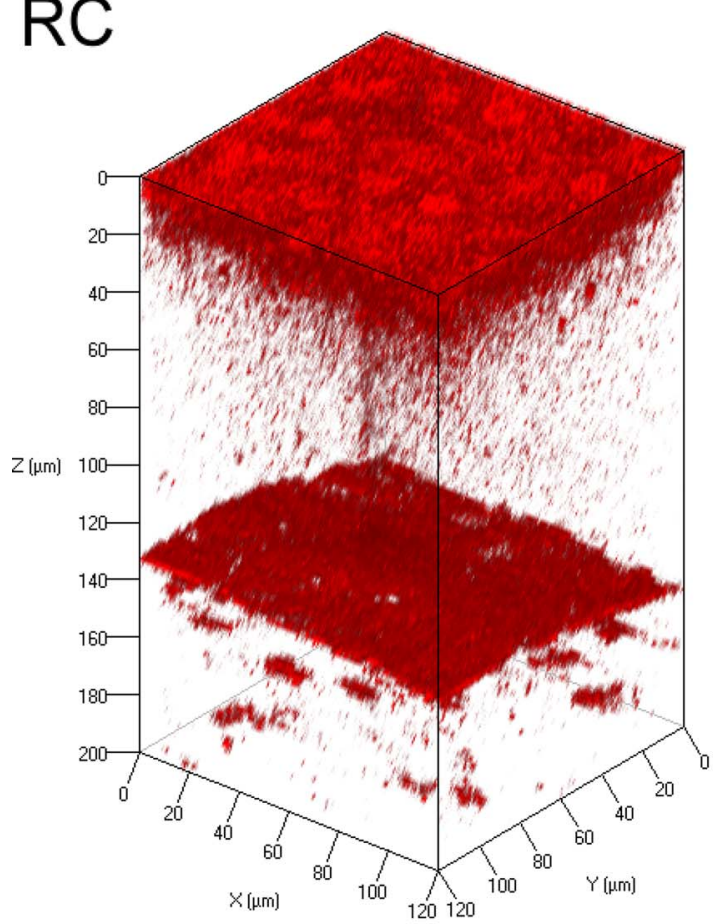

(a)

\section{SHG}

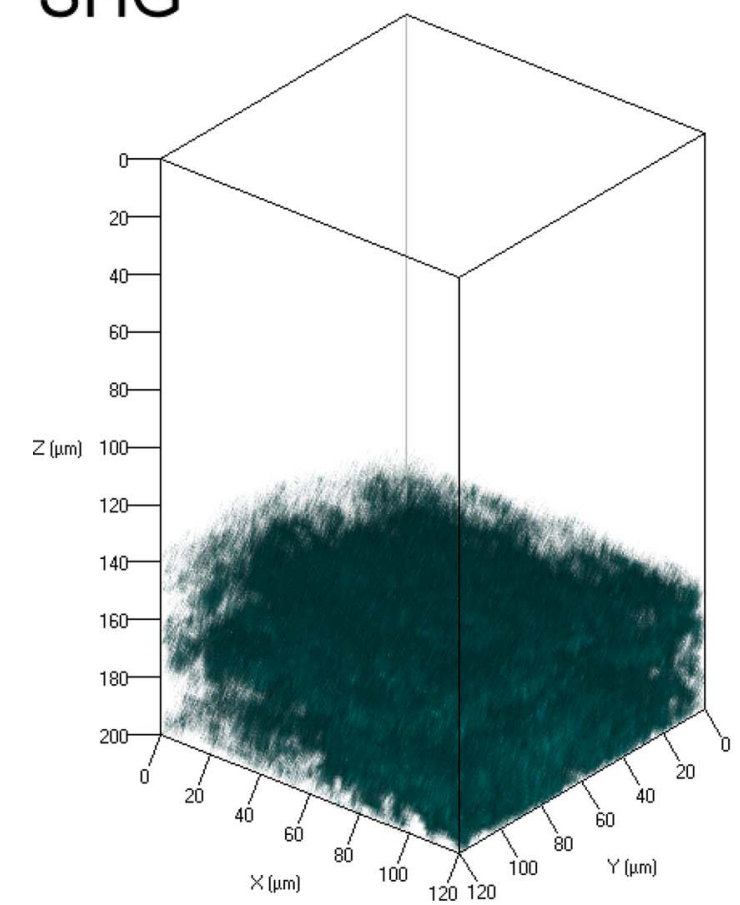

(c)

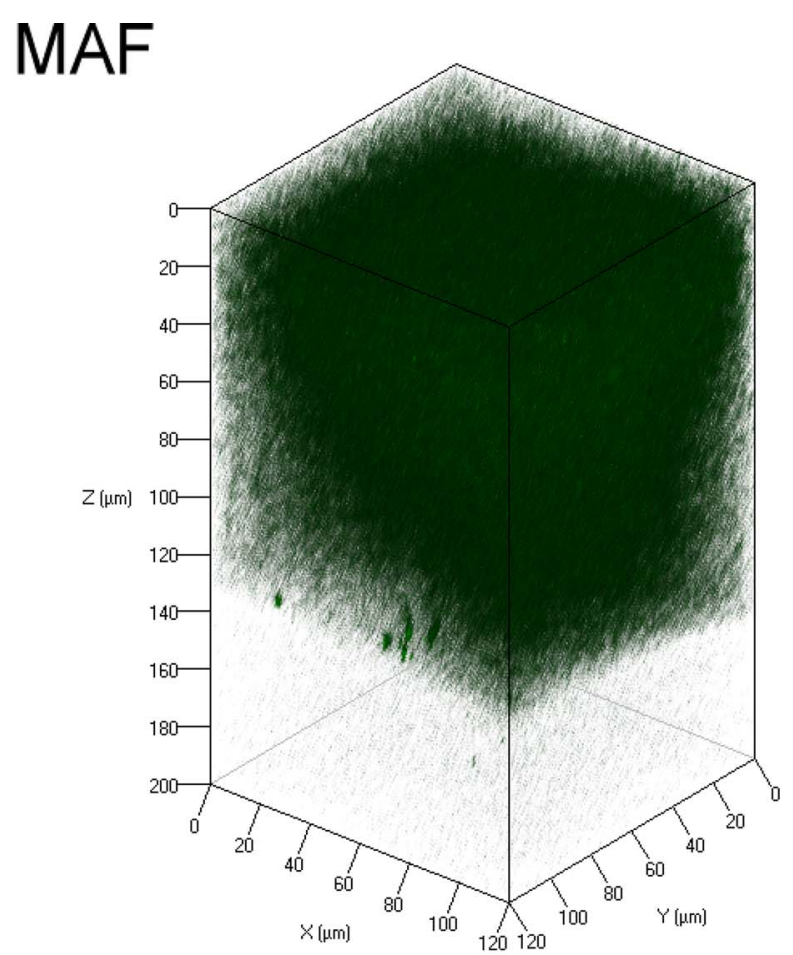

(b)

\section{Combined}

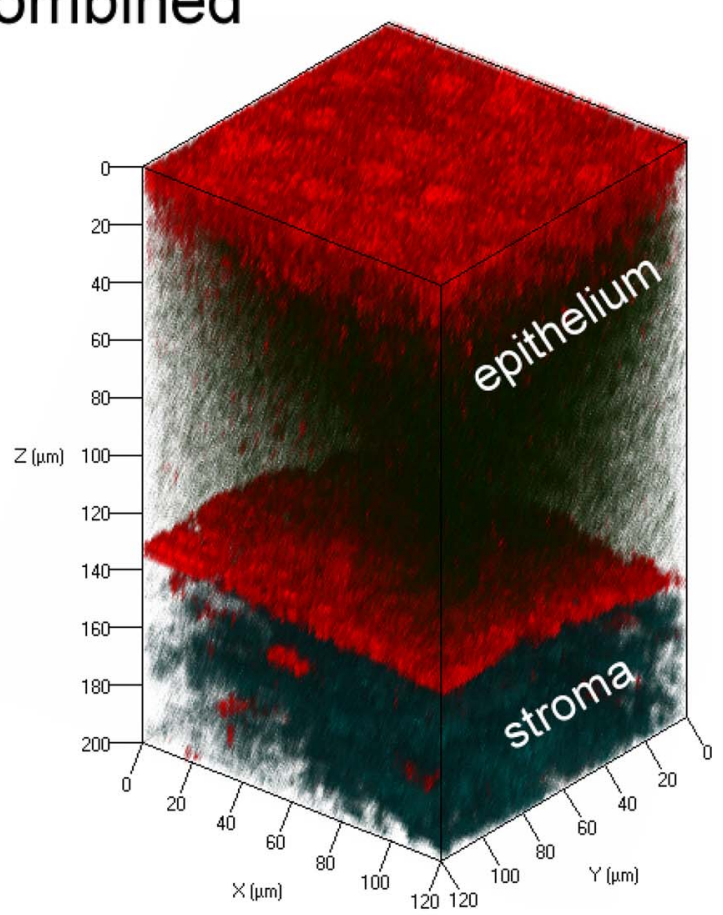

(d)

Fig. 5 (a) RC (red), (b) MAF (green), (c) SHG (cyan), and (d) combined RC, MAF, and SHG imaging of the ex vivo bovine cornea specimen in three dimensions. Image sizes are $115 \times 115 \times 200 \mu \mathrm{m}^{3}$. (Color online only.)

200 and $100 \mu \mathrm{m}$, respectively. Similarly, the MAF signal can be detected to approximately the same depths. However, note that, as a function of depth, the detected MAF signal decays at a slower rate than the RC signal, suggesting that the MAF imaging is a preferred imaging modality for probing into deeper layers of the skin and corneal epithelia. Furthermore, in the corneal RC signal profile, a peak exists at about $140 \mu \mathrm{m}$, corresponding to the Bowman's layer in the epithelium-stroma junction. A similar peak was not found for the skin, suggesting that a sharper refractive change exists at 


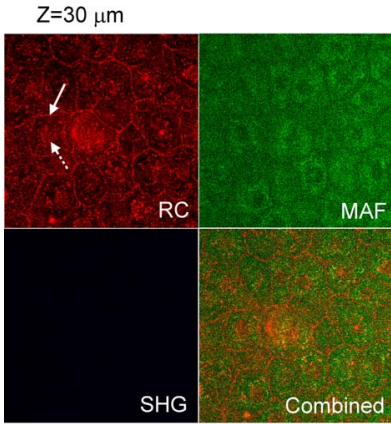

(a)

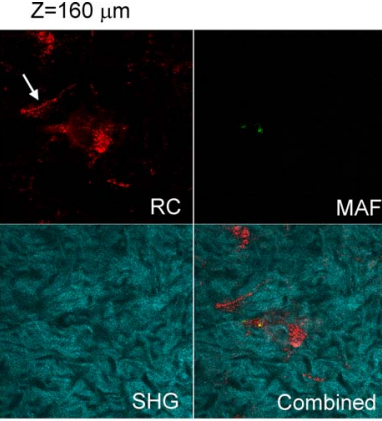

(b)
Fig. 6 RC (red), MAF (green), SHG (cyan), and combined RC, MAF, and SHG imaging of the ex vivo bovine cornea in two dimensions. Representative images at the approximate depths of (a) $30 \mu \mathrm{m}$ and (b) $160 \mu \mathrm{m}$ are shown. Image sizes are $115 \times 115 \mu \mathrm{m}^{2}$. (Color online only.)

the corneal epithelium-stroma junction than that at the skin epithelial-dermal junction. In addition, in both the skin and cornea specimens, the SHG signal rises sharply beyond the epithelium, indicating the effectiveness of SHG imaging for probing the collagen matrix. Nonetheless, one should keep in mind that the nature of signal generation is different for the $\mathrm{RC}, \mathrm{MAF}$, and SHG signals. The RC signal occurs at interfaces of different refractive indices, while the MAF intensity depends on the concentration of autofluorescent molecules present. Quantification of the SHG signal also depends on the amount of noncentrosymmetric biomolecular structures present.

The comparision of 780-, 488-, 543-, and 633-nm RC imaging on a porcine skin sample and a cornea sample are made, respectively, in Figs. 8(a) and 8(b). The skin images shown were acquired at the depth of $15 \mu \mathrm{m}$, and the cornea images were acquired at the depth of $30 \mu \mathrm{m}$. From the RC images, we see only slight or no image degradation with increasing wavelength. To investigate the relative change in RC intensity with increasing imaging depth, we plot the normalized RC intensity as a function of depth for the porcine skin [Fig. 9(a)] and bovine cornea [Fig. 9(b)] samples. From Fig. 9(b), we see that the RC intensity in bovine cornea imaging decays more slowly with increasing laser wavelength, as expected, since longer wavelength light is less susceptible to scattering by

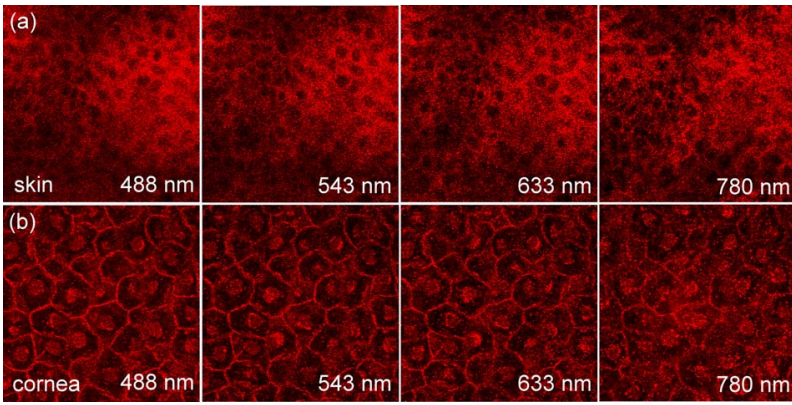

Fig. 8 RC imaging using four different laser light wavelengths (488, 543,633 , and $780 \mathrm{~nm}$ ) for (a) porcine skin at a depth of $15 \mu \mathrm{m}$ and (b) bovine cornea specimen at a depth of $30 \mu \mathrm{m}$. Image sizes are $115 \times 115 \mu \mathrm{m}^{2}$.

tissue sample. However, for porcine skin RC imaging, we found that the RC signal decays at a slower rate at shorter wavelengths. One possible explanation is that the porcine skin tissue sample we used preferentially absorbs at longer wavelength, despite the fact that less scattering occurs at longer wavelength. Furthermore, since the detected reflectance confocal signals can come from out of the focal plane sample scattering, the value of $I / I_{0}$ may not accurately quantify the contrast provided by reflectance confocal imaging. This effect is particular significant in highly scattering tissues such as skin. Figure 10 shows the reflected confocal images of porcine skin at the depth of $40 \mu \mathrm{m}$ for the four wavelengths. By visual inspection, we see that increasing wavelength does indeed lead to better image contrast, despite having a lower $I / I_{0}$ ratio. These findings suggest that although a shorter wavelength provides slightly better image quality near the surface, longer wavelengths are more effective for in-depth RC imaging.

In addition to investigating wavelength dependence of $\mathrm{RC}$ imaging, we also investigated the effect of laser intensity for $780-n m$ RC imaging. Figure 11 shows RC image of areas in the skin and cornea sample using 780-nm light of varying powers (on sample). We found that within the power range tested, increasing the power does not lead to any observable improvements in the image quality. Since optimal power for MAF and SHG imaging typically requires on-sample power of greater than a few milliwatts, the optimal power for simul-

\section{skin}

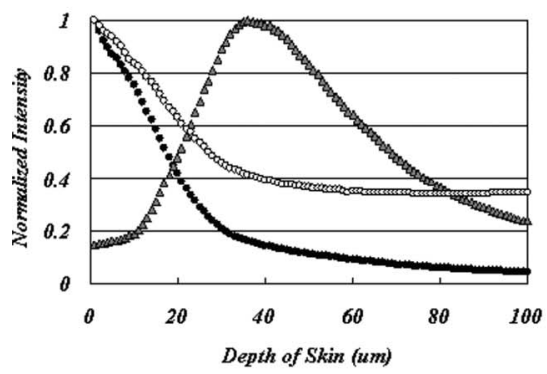

(a)

\section{cornea}

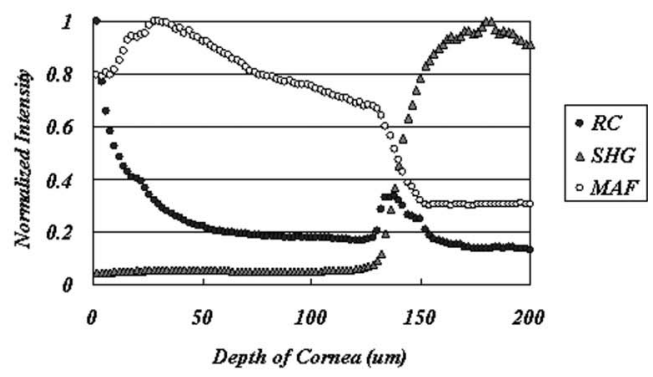

(b)

Fig. 7 Measured RC, MAF, and SHG signal strengths as a function of imaging depths from the (a) ex vivo porcine skin and (b) ex vivo bovine cornea specimens. 
skin

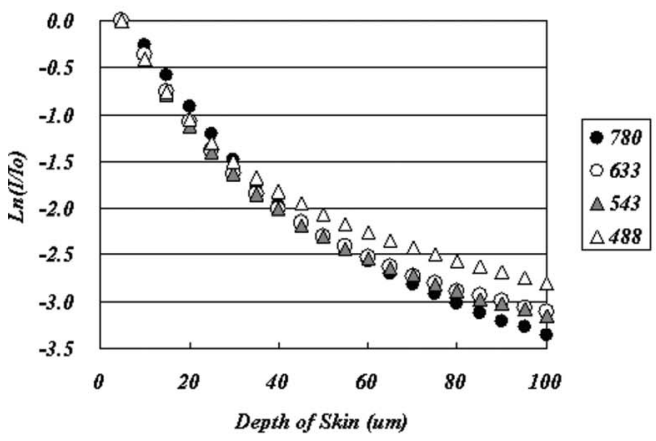

(a) cornea

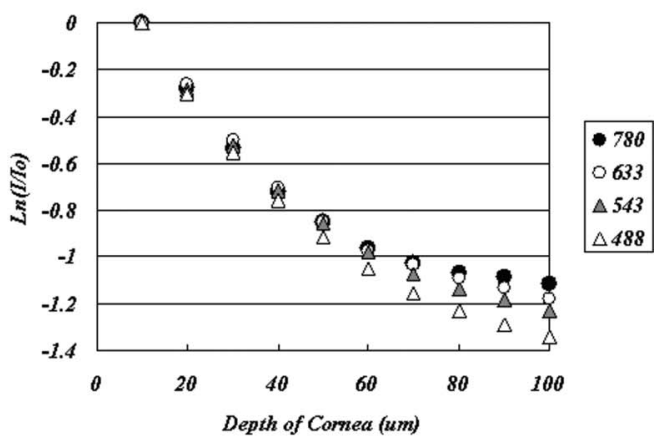

(b)

Fig. 9 Semilog plot of the wavelength dependence of normalized RC intensity as a function of imaging depth for (a) porcine skin and (b) bovine cornea specimen.

taneous RC, MAF, and SHG imaging is dictated by the requirement for multiphoton imaging. In our study, the imaging power is limited by the threshold for tissue photodamage due to multiphoton excitation.

\section{Conclusions}

We demonstrated RC, MAF, and SHG imaging using a single excitation wavelength of $780 \mathrm{~nm}$. In both the ex vivo specimens of porcine skin and bovine cornea, the near-IR, RC signal was effective for imaging the cellular boundaries of the epithelia. However, in the case of porcine skin, additional RC signals from within the intracellular regions enabled additional structural imaging. In both cases, nuclei can be detected from the RC signal. On the other hand, the MAF signal can be

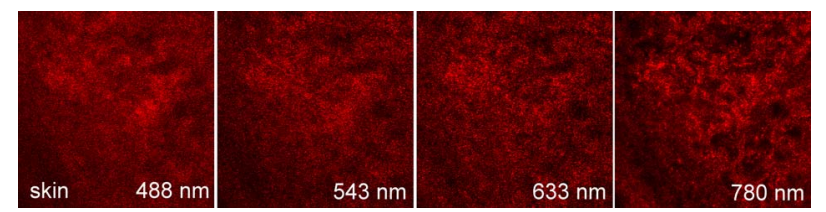

Fig. 10 RC imaging using four different laser light wavelengths (488, 543,633 , and $780 \mathrm{~nm}$ ) for porcine skin at a depth of $40 \mu \mathrm{m}$. Image sizes are $115 \times 115 \mu \mathrm{m}^{2}$. Visible image contrast improvements are observed with increasing wavelength.

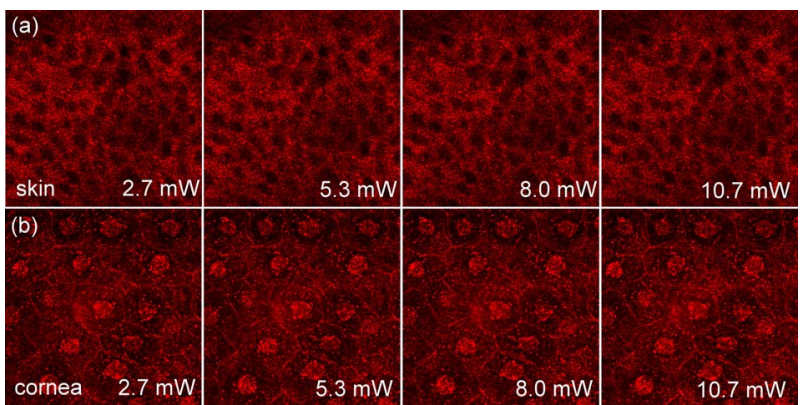

Fig. 11 RC imaging of (a) porcine skin and (b) bovine cornea using different laser powers at $780 \mathrm{~nm}$. Image sizes are $115 \times 115 \mu \mathrm{m}^{2}$. The corresponding on-sample laser power is shown on each image. No significant difference in image quality is observed for RC imaging in the power range tested. used for cytoplasmic imaging through the entire epithelium. Since broadband AF signal (435 to $700 \mathrm{~nm}$ ) was collected, identification of the molecular species contributing to the MAF signal was not made. However, a main contributor to the MAF signal is ${ }^{8}$ most likely NAD $(\mathrm{P}) \mathrm{H}$. Beyond the epithelia, SHG microscopy can be applied for characterizing the underlying collagen matrix with the RC signal capable of identifying individual corneal keratocytes. We found that the use of longer 780-nm femtosecond pulse laser for deep RC imaging of bovine cornea and porcine skin resulted in images with visibly improved image contrast than the images acquired from using continuous wave laser sources at wavelengths of 488, 543, and $633 \mathrm{~nm}$. Furthermore, varying the $780-\mathrm{nm}$ power for RC imaging produced images with comparable quality. Our results demonstrate that with the appropriate instrument, it is possible to achieve simultaneous RC, MAF, and SHG imaging using a single femtosecond excitation source. The contrast offered by this multimodal approach may be extended for the clinical diagnosis of diseases in the future.

\section{Acknowledgments}

This study was supported in part by the National Research Program for Genomic Medicine (NRPGM) of the National Science Council (NSC) of Taiwan (NSC93-3112-B-002-033 and NSC93-3112-B-002-034, and NSC-95-3112-B-002-019), and was completed in the Optical Molecular Imaging Microscopy Core Facility (A5) of NRPGM.

\section{References}

1. M. Rajadhyaksha, M. Grossman, D. Esterowitz, R. H. Webb and R. $\mathrm{R}$. Anderson, "In vivo confocal scanning laser microscopy of human skin-melanin provides strong contrast," J. Invest. Dermatol. 104(6), 946-952 (1995).

2. M. Rajadhyaksha, M. Grossman, R. H. Webb, and R. R. Anderson, "Video-rate confocal scanning laser microscopy of live human skin," in Abstracts, 1995 Ann. Meeting of the Society for Investigative Dermatology, J. Invest. Dermatol. 104(4), 618 (1995).

3. J. V. Jester, P. M. Andrews, W. M. Petroll, M. A. Lemp, and H. D. Cavanagh, "In vivo, real-time confocal imaging," J. Electron $\mathrm{Mi}$ crosc. Tech. 18(1), 50-60 (1991).

4. B. R. Masters and M. Bohnke, "Three-dimensional confocal microscopy of the living human eye," Annu. Rev. Biomed. Eng. 4, 69-91 (2002). 
5. P. T. C. So, C. Y. Dong, B. R. Masters, and K. M. Berland, "Twophoton excitation fluorescence microscopy," Аnnu. Rev. Biomed. Eng. 2, 399-429 (2000).

6. S. W. Chu, S. P. Tai, C. L. Ho, C. H. Lin, and C. K. Sun, "Highresolution simultaneous three-photon fluorescence and thirdharmonic-generation microscopy," Microsc. Res. Tech. 66(4), 193197 (2005).

7. P. J. Campagnola and L. M. Loew, "Second-harmonic imaging microscopy for visualizing biomolecular arrays in cells, tissues and organisms," Nat. Biotechnol. 21(11), 1356-1360 (2003).

8. W. R. Zipfel, R. M. Williams, R. Christie, A. Y. Nikitin, B. T. Hyman, and W. W. Webb, "Live tissue intrinsic emission microscopy using multiphoton-excited native fluorescence and second harmonic generation," Proc. Natl. Acad. Sci. U.S.A. 100(12), 7075-7080 (2003).

9. B. R. Masters, P. T. C. So, and E. Gratton, "Multiphoton excitation fluorescence microscopy and spectroscopy of in vivo human skin," Biophys. J. 72(6), 2405-2412 (1997).

10. A. T. Yeh, N. Nassif, A. Zoumi, and B. J. Tromberg, "Selective corneal imaging using combined second-harmonic generation and two-photon excited fluorescence," Opt. Lett. 27(23), 2082-2084 (2002).

11. S. W. Teng, H. Y. Tan, J. L. Peng, H. H. Lin, K. H. Kim, W. Lo, Y. Sun, W. C. Lin, S. J. Lin, S. H. Jee, P. T. C. So, and C. Y. Dong, "Multiphoton autofluorescence and second-harmonic generation imaging of the ex vivo porcine eye," Invest. Ophthalmol. Visual Sci. 47(3), 1216-1224 (2006).
12. S. J. Lin, R. J. Wu, H. Y. Tan, W. Lo, W. C. Lin, T. H. Young, C. J. Hsu, J. S. Chen, S. H. Jee, and C. Y. Dong, "Evaluating cutaneous photoaging by use of multiphoton fluorescence and second-harmonic generation microscopy," Opt. Lett. 30(17), 2275-2277 (2005).

13. S. J. Lin, S. H. Jee, C. J. Kuo, R. J. Wu, W. C. Lin, J. S. Chen, Y. H. Liao, C. J. Hsu, T. F. Tsai, Y. F. Chen, and C. Y. Dong, "Discrimination of basal cell carcinoma from normal dermal stroma by quantitative multiphoton imaging," Opt. Lett. 31(18), 2756-2758 (2006).

14. R. Cicchi, D. Massi, S. Sestini, P. Carli, V. De Giorgi, T. Lotti, and F. S. Pavone, "Multidimensional non-linear laser imaging of basal cell carcinoma," Opt. Express 15(16), 10135-10148 (2007).

15. M. Han, L. Zickler, G. Giese, M. Walter, F. H. Loesel, and J. F. Bille, "Second-harmonic imaging of cornea after intrastromal ferntosecond laser ablation," J. Biomed. Opt. 9(4), 760-766 (2004).

16. H. Y. Tan, Y. Sun, W. Lo, S. J. Lin, C. H. Hsiao, Y. F. Chen, S. C. M. Huang, W. C. Lin, S. H. Jee, H. S. Yu, and C. Y. Dong, "Multiphoton fluorescence and second harmonic generation imaging of the structural alterations in keratoconus ex vivo," Invest. Ophthalmol. Visual Sci. 47(12), 5251-5259 (2006).

17. H. Y. Tan, Y. Sun, W. Lo, S. W. Teng, R. J. Wu, S. H. Jee, W. C. Lin, C. H. Hsiao, H. C. Lin, Y. F. Chen, D. H. K. Ma, S. C. M. Huang, S. J. Lin, and C. Y. Dong, "Multiphoton fluorescence and second harmonic generation microscopy for imaging infectious keratitis," $J$. Biomed. Opt. 12(2), 024013 (2007).

18. W. L. Chen, Y. Sun, W. Lo, H. Y. Tan, and C. Y. Dong, "Combination of multiphoton and reflective confocal imaging of cornea," Microsc. Res. Tech. 71(2), 83-85 (2008). 